Научная статья

УДК ОО $4+658$

DOI: $10.17213 / 2075-2067-2021-5-156-170$

\title{
КАК ОЦЕНИТЬ И МИНИМИЗИРОВАТЬ ЗАТРАТЫ ВРЕМЕНИ И ФИНАНСОВЫХ РЕСУРСОВ НА ПРОЦЕССЫ ОБУЧЕНИЯ ГРАЖДАН: МЕТОДЫ И ИНСТРУМЕНТЫ
}

\author{
Георгий Николаевич Хубаев \\ Ростовский государственный экономический университет (РИНХ), \\ Ростов-на-Дону, Россия \\ gkhubaev@mail.ru, ORCID: 0000-0003-1986-926Х, AuthorID РИНЦ: 154667
}

Аннотация. Целью исследования является разработка методики, основанной на использовании совокупности апробированных методов и инструментальных средств, для оценки и минимизации затрат времени и финансовых ресурсов на обучение граждан.

Методологическую базу исследования представляют экономическая теория, статистические методы, экономико-математические методы и модели.

Результаты исследования. Выявлены и систематизированы существующче проблемы и трудности, содержательно обоснованы исходные условия и критерии оценки качества обучения, предложены апробированные методы и инструментальные средства для сравнительной оценки характеристик потребительского качества различных видов и средств методического, кадрового и инструментального обеспечения учебных процессов, для оценки и минимизации затрат времени и финансовых ресурсов государства и юридчческих лич - субъектов рынка на обучение граждан.

Перспектива исследования состочт в том, что предложенная совокупность методов и инструментальных средств, подтвердивших в проиессе исследований прикладную полезность, применима ${ }^{*}$ для оценки и минимизаџии затрат финансовых ресурсов государства и юридических лич - субъектов рынка на обучение граждан и "для сравнительной оченки характеристик потребительского качества методического, кадрового и инструментального обеспечения учебных прочессов в различных образовательных учреждениях и предметных областях.

Ключевые слова: учебный прочесс, методическое обеспечение, инструментальные средства, потребительское качество, критерии оченки, образовательный стандарт, интердецильный размах, статистические методы

Для цитирования: Хубаев Г.Н. Как оценить и минимизировать затраты времени и финансовых ресурсов на прочессы обучения граждан: методы и инструменты // Вестник Южно-Российского государственного технического университета. Серия: Социальноэкономические науки. 2021. T. 14, №5. C. 156-170. http://dx.doi.org/10.17213/2075-2067-20215-156-170.

(C) Хубаев Г.Н., 2021 
Original article

\title{
HOW TO EVALUATE AND MINIMIZE THE TIME AND FINANCIAL RESOURCES SPENT ON CITIZEN EDUCATION PROCESSES: METHODS AND TOOLS
}

\author{
Georgy N. Khubaev \\ Rostov State University of Economics (RSUE), Rostov-on-Don, Russia \\ gkhubaev@mail.ru, ORCID: 0000-0003-1986-926X, AuthorID RSCI: 154667
}

\begin{abstract}
The purpose of the study is to develop a methodology based on the use of a set of proven methods and tools to assess and minimize the time and financial resources spent on training citizens.

The methodological basis of the research is economic theory, statistical methods, economic and mathematical methods and models.

The results of the study: the existing problems and difficulties are identified and systematized, the initial conditions and criteria for assessing the quality of training are substantiated in a meaningful way, proven methods and tools are proposed for a comparative assessment of the characteristics of consumer quality of methods and means of methodological, personnel and instrumental support of educational processes, for assessing and minimizing the time and financial resources of the state and legal entities - subjects of the market for training citizens.

The perspective of the study is that the proposed set of methods and tools, which have confirmed their applied usefulness in the course of research, is applicable *to assess and minimize the costs of financial resources of the state and legal entities - market entities for training citizens and "for a comparative assessment of the characteristics of the consumer quality of methodological, personnel and instrumental support of educational processes in various educational institutions and subject areas.
\end{abstract}

Keywords: educational process, methodological support, tools, consumer quality, evaluation criteria, educational standard, interdecile scope, statistical methods

For citation: Khubaev G. N. How to evaluate and minimize the time and financial resources spent on citizen education processes: methods and tools // Bulletin of the South Russian State Technical University. Series: Socio-economic Sciences. 2021; 14(5): 156-170. (In Russ.). http:// dx.doi.org/10.17213/2075-2067-2021-5-156-170.

Введение. С развитием научно-технического прогресса (НТП) все меньшую часть актуальной информации может освоить даже очень ответственный и способный человек. Так, в современных условиях из-за непрерывного ускорения НТП, уменьшения периода усвоения знаний, лавинообразного роста числа публикаций по большинству направлений науки и техники полученные ранее знания быстро теряют актуальность, устаревают. Причем в последние годы ускорение НТП неизбежно *приводит правительство и население страны к осознанию необходимос- ти ускорять, интенсифицировать процессы обучения и одновременно *заставляет задуматься о причинах роста затрат на получение нужного уровня (объема) знаний, умений, навыков, компетенций, * побуждает и зачастую вынуждает граждан учиться всю сознательную жизнь. При этом оказалось, что чем больше в генеральной совокупности, именуемой населением страны, доля талантливых и компетентных профессионалов, делающих новые и полезные для граждан разработки и открытия, тем быстрее растет экономика страны и, как следствие, уровень жизни насе- 
ления. И поэтому становится очевидной необходимость добиваться того, чтобы процесс выявления, обучения и поддержки новых Ломоносовых, Ньютонов, Канторовичей, Колмогоровых и множества других гигантов науки не был случайным, а стал бы целенаправленным, непрерывным, квази-детерминированным, с минимальным количеством ошибок.

В такой ситуации задача поиска методов и инструментов, позволяющих оценивать и минимизировать затраты времени и финансовых ресурсов на обучение индивида в любой сфере его жизнедеятельности, представляется исключительно актуальной. Одновременно внимание общества к процессам обучения граждан вынуждает и организации, специализирующиеся на предоставлении образовательных услуг, также заниматься поиском возможностей снизить затраты времени и финансовых ресурсов обучаемых на получение знаний.

В статье на примере обучения студентов в вузе выявлены и систематизированы существующие проблемы и трудности, содержательно обоснованы исходные условия и критерии оценки качества процессов обучения, предложены апробированные методы и инструментальные средства для сравнительной оценки характеристик потребительского качества видов и средств методического и инструментального обеспечения учебных процессов, для оценки и минимизации затрат времени и финансовых ресурсов на обучение граждан.

При этом описанные методы и инструментальные средства применимы для сравнительной оценки потребительского качества методического, кадрового и инструментального обеспечения учебных процессов в различных предметных областях, для оценки и минимизации затрат финансовых ресурсов государства и юридических лиц-субъектов рынка на обучение граждан.

\section{1. Существующие проблемы и трудности}

1) В связи с тем, что многие граждане вынуждены учиться всю жизнь, обучаемые могут быть разного возраста, с разным уровнем и разной профессиональной направленностью образования (техники и гуманитарии, «физики и лирики»), с разными психофизиологическими и физическими особенностями.
Однако неизвестно, какие факторы влияют * на скорость получения знаний в целом, независимо от внешних и внутренних условий протекания учебного процесса, "на продолжительность освоения установленного объема знаний в случае неоднородной совокупности обучаемых, и "какова степень этого влияния.

Как, какими методами, корректно или не очень, с определенной, заранее известной степенью достоверности все это оценить и использовать для оптимальной организации учебных процессов, для минимизации затрат времени и финансовых ресурсов на обучение граждан?

2) В настоящее время отсутствуют обязательные в масштабах всей страны, единые для всех (обучаемых и преподавателей) варианты контрольных испытаний - экзаменационных задач, перечни проблемных ситуаций.

Но как в таких условиях можно быть уверенным в том, что в разных городах и в разных административно-территориальных образованиях (АТО) все студенты, получившие на экзамене, например, отличные оценки, достигли одинакового, установленного образовательным стандартом уровня знаний, обладают установленными стандартом компетенциями?

3) В образовательных стандартах и учебных планах специальностей перечислены учебные дисциплины, указано время, в течение которого должна изучаться каждая дисциплина, представлены компетенции, которыми должен обладать обучаемый.

Однако не указано, *как осуществлялось формирование образовательного стандарта, *как выполнялось ранжирование компетенций при формировании специальностей и выделении компетенций для каждой специальности, "насколько обоснованы отведенные на изучение дисциплин отрезки времени, "какова допустимая величина взаимосвязи по компетенциям и между дисциплинами, и между специальностями, и *как, каким методом это все оценивалось. Но ведь в таком случае невозможно определить, каков информационный вес той или иной компетенции или дисциплины, какова степень взаимосвязи по компетенциям между дисциплинами и модулями одной специальности и т.д. 
Но определить, рассчитать все это совсем не просто. Действительно, ведь только базовая (обязательная) часть образовательного стандарта содержит десятки дисциплин, в результате изучения которых студент должен получить знания, умения, навыки и сформировать 30-50 компетенций. Но и вариативная часть также содержит десятки дисциплин и множество модулей, обеспечивающих получение студентом необходимых компетенций, предусмотренных государственным образовательным стандартом.

Так, анализ содержания государственных образовательных стандартов по ряду специальностей высшего профессионального образования (квалификация «бакалавр», специальности: 080500 — Бизнес-информатика, 230700 - Прикладная информатика, 90900 - Информационная безопасность и др.) показал, что если каждая дисциплина содержит 3-4 модуля, а дисциплин в среднем 40-50, то в учебном плане будет 100-200 модулей. В свою очередь, каждая из 30-50 компетенций включает 3-5 компонентов (составляющих). Тогда общее количество компонентов, формирующих компетенции, будет находиться в пределах 100-250.

Спрашивается, как определить, полноту охвата содержанием дисциплин всей совокупности компетенций?

Как количественно определить, насколько удачно сформирована вариативная часть учебного плана?

В какой степени десятки включенных в учебный план дисциплин (и множество модулей) обеспечивают возможность получения студентом множества необходимых компетенций? И какими дисциплинами или модулями такие возможности лучше обеспечиваются, а какими - хуже и насколько?

Вряд ли можно надеяться на то, что ктонибудь из обычных людей сможет ответить на эти вопросы.

4) По аналогии с технологическими и управленческими процессами для улучшения характеристик качества учебного процесса формируется его методическое, кадровое и инструментальное обеспечение, включающее учебники, кадровый состав, компьютерные обучающие системы, образовательные стандарты и учебные планы, инструкции для покупателей многофункциональных то- варов и товаров со сложной структурой или многокомпонентным составом, программы повышения квалификации работающих и множество других помощников обучаемых и работников учреждений, предоставляющих образовательные услуги. И именно степень влияния этих помощников на оперативность и ресурсоемкость процессов обучения граждан характеризует потребительское качество методического, кадрового и инструментального обеспечения учебного процесса.

Причем оказалось, что уже сформировано множество одинаковых по назначению, но разных по содержанию и цене методических и инструментальных средств: разных по структуре и содержанию учебников для освоения одной и той же дисциплины учебного плана, множество компьютерных систем для обучения, тестирования и систем обеспечения информационной безопасности, методов, ориентированных на помощь в понимании и запоминании новой информации, и множество других видов обеспечения учебного процесса.

Как будто все это хорошо в условиях рыночной экономики - конкуренция.

Но, спрашивается, как при такой ситуации потенциальному ученику - субъекту рыночных отношений или даже руководителю образовательного учреждения - разобраться с таким множеством помощников? Ведь неизвестно, как эти разные помощники одного назначения влияют на характеристики потребительского качества данного вида обеспечения учебного процесса, для эффективной реализации которого они и созданы.

Как определить, с использованием каких методов и инструментов следует оценивать и количественно сравнивать потребительское качество инструментария, обеспечивающего и формирующего учебный процесс?

И самое главное - как определять, какой из такого множества помощников обеспечивает более высокое по сравнению с конкурентами качество выполнения учебного процесса, получение обучаемыми хороших знаний, умений, навыков и компетенций с минимальными затратами времени и других ресурсов?

Сегодня нет ответа на эти вопросы.

Очевидно, что разное потребительское качество отдельных видов и средств мето- 
дического, кадрового и инструментального обеспечения учебного процесса, разная их цена и степень влияния на величину затрат времени и финансовых ресурсов на обучение граждан одновременно приводит и к разной результативности учебного процесса, к разным затратам времени индивида на получение одинакового объема знаний, умений, навыков, компетенций.

И то, что в реальности НИКТО не знает, как влияет на величину затрат времени и финансовых ресурсов и государства, и вузов, и обучаемых использование в процессе обучения разных учебников, разных компьютерных обучающих систем, разных учебных планов, разных оценочных материалов и систем, разных методов обучения, приводит к тому, что и выпускники одинаковых специальностей, но разных вузов, в разных городах одной страны, в разных административно-территориальных образованиях имеют разный уровень (объем) знаний, умений, навыков, компетенций.

\section{2. Исходные условия и критерии оцен- ки качества обучения}

1) Методическое, кадровое и инструментальное обеспечение учебного процесса - это учебники, преподаватели, компьютерные обучающие, тестирующие и обеспечивающие информационную безопасность системы, используемые в учебном процессе лицензионные программные продукты и информационные системы, системы, выполняющие сложные вспомогательные расчеты, методы, ориентированные на помощь в понимании и запоминании новой информации, и множество других видов и средств, обеспечивающих процессы обучения граждан.

Основной характеристикой потребительского качества каждого вида методического, кадрового и инструментального обеспечения учебного процесса служит то, насколько этот конкретный обеспечивающий объект (вид, средство) снижает затраты времени обучаемых на овладение требуемым составом компетенций и какова его цена при прочих равных условиях (затратах времени на освоение, стоимость доставки и др.).

2) Условимся, что обучаемых можно разделить на две группы: *лица примерно одного возраста, уровня исходного, базового образования, одинаковой заинтересованности в освоении новой учебной дисциплины и т.д., т.е. совокупность достаточно однородных объектов, и "лица разного возраста, разного уровня образования, разной степени заинтересованности в результатах обучения и с разными психофизиологическими и физическими особенностями.

3) Сравнительная количественная оценка потребительского качества методического, кадрового и инструментального обеспечения учебного процесса (УП) и оценка затрат времени обучаемых на овладение знаниями базируются на использовании статистических методов.

Так, при сравнении потребительского качества разных видов и средств обеспечения учебного процесса используется интердецильный размах, практически не зависящий от экстремальных значений в выборке (появляется возможность исключить или уменьшить влияние неоднородности в составе обучаемых) и одновременно включающий основную часть выборки и, как недавно подтверждено также и нашими исследованиями, позволяющий иметь очень малые колебания стандартного отклонения в выборках из одной и той же генеральной совокупности. В такой ситуации появляется возможность с большей уверенностью использовать треугольное распределение при имитационном моделировании процессов. А если, например, при экспериментальных исследованиях или при обработке результатов опроса студентов разных учебных групп о затратах времени на подготовку к экзаменам при использовании учебников У1 и У2 (разных авторов) получено две независимых выборки: одна с ответами студентов, которые готовились к экзамену, используя учебник У1, а вторая - с использованием учебника У2? В таком случае необходимо ответить на вопpoc: относятся ли эти выборки к одной генеральной совокупности? Или иначе: можно ли считать, что подготовка к экзамену с использованием одного из конкурирующих учебников более предпочтительна, дает лучшие результаты? Выборки относятся к разным генеральным совокупностям. Для реализации такой проверки статистически значимых различий потребительского качества учебников У1 и У2 используется наиболее строгий кри- 
терий однородности: критерий Колмогорова и Смирнова - нуль гипотеза (генеральные совокупности одинаковы) против альтернативной гипотезы (генеральные совокупности имеют различные распределения).

4) Доля специалистов, обладающих высоким уровнем знаний и умений в любой стране и в любой сфере профессиональной деятельности, невелика (лишь 10-20\%). И даже незначительное увеличение этой доли исключительно актуально. Для оценки объема и уровня полученных в процессе обучения знаний в статье используются статистические характеристики распределения показателя «Затраты времени на решение задач», разработанных подмножеством компетентных специалистов (математическое ожидание, коэффициент вариации, асимметрия, эксцесс, интердецильный размах), или показателя «Количество решенных за определенное, заданное время задач».

Содержание таких задач связано с изученным учебным предметом, а решение направлено на улучшение (реальных или придуманных разработчиками) проблемных ситуаций в анализируемой конкретной предметной области или в сфере конкретной профессиональной деятельности.

5) Люди очень разные *по способностям, которыми их наделила природа, и по "психофизиологическим и физическим особенностям, обусловленным жизненным опытом. Естественно, что все эти особенности в той или иной степени влияют на затраты времени обучаемого при овладении им компетенциями, которые заданы образовательным стандартом, т.е. эти отличия влияют на скорость, оперативность восприятия новой информации, на величину необходимого обучаемому промежутка времени для ее усвоения, на осознание прикладной полезности новых сведений. И чем сложнее новая информация, тем эта разница будет заметнее: величина правосторонней асимметрии у группы обучаемых растет очень быстро. Поэтому при оценке затрат времени на изучение новой информации, новых учебных дисциплин, при сравнительной оценке потребительского качества отдельных видов методического и инструментального обеспечения учебного процесса мы ориентируемся именно на интердецильный размах, поскольку наш опыт свидетельствует о том, что в первый дециль попадают люди, обладающие и способностями, и целеустремленностью, старательностью, в последний - те, кому перестала нравиться учебная дисциплина или возникли трудности, обусловленные множеством других причин.

\section{3. Использованные методы и инстру-} ментальные средства. Для элиминирования перечисленных ранее проблем и трудностей, с которыми сталкиваются в вузах и других образовательных учреждениях, выполнен анализ возможности и обоснованности использования различных методов и инструментальных средств: "для формирования состава разработчиков оценочных материалов, позволяющих определять степень наличия у обучаемого определенного (заданного) уровня знаний, "для ранжирования и обоснованного выбора состава компетенций при формировании специальностей и учебных планов, "для экспериментальной сравнительной оценки затрат времени обучаемых на изучение новых материалов и оценки потребительского качества разных видов и средств обеспечения учебного процесса, "для экспертной оценки необходимого и достаточного времени на изучение дисциплин, представленных в учебных планах специальностей.

3.1. Формирование состава разработчиков оценочных материалов - экзаменационных задач и перечня проблемных ситуаций. Формирование состава компетентных разработчиков оценочных материалов, предназначенных для определения степени владения обучаемым содержанием изученной учебной дисциплины, - это главное условие для получения обоснованной оценки потребительского качества методического, кадрового и инструментального обеспечения учебного процесса, для оценки степени влияния его отдельных видов на эффективность учебного процесса, на степень овладения обучаемым заданным составом компетенций. В качестве претендентов на разработку экзаменационных задач и проблемных ситуаций, помимо преподавателей вузов, которые ведут занятия по рассматриваемой учебной дисциплине, могут быть авторы различных видов методического и инструментального обеспечения учебного процесса. 
Но реализация процесса формирования и выбора состава разработчиков оценочных материалов представляет собой достаточно сложную проблему.

Какие же трудности возникают при реализации процедуры выбора? Во-первых, очевидно, что разработку и экспертизу составленных задач и проблемных ситуаций должны выполнять компетентные, талантливые, ответственные, заинтересованные в оптимальном решении поставленной задачи люди. Но таких людей необходимо еще найти! Во-вторых, сегодня нет однозначного понимания того, какой должна быть сама процедура оценки правильности, объективности, эффективности того или иного способа решения этой задачи. Так возникает вторая трудность - это поиск процедуры формирования коллективного выбора в процессе подготовки оценочных материалов.

Кроме того, трудно надеяться на то, что потенциальные разработчики, не будучи студентами, согласятся решать составленные для них задачи. В такой ситуации единственно возможным способом сформировать подмножество разработчиков оценочных материалов для проведения контрольных испытаний лиц, завершивших обучение по конкретному предмету или по теме повышения квалификации, является ориентация на методы ранжирования объектов по количественным показателям, например, по таким широко используемым, как «Количество опубликованных работ по определенному направлению», «Количество цитирований», наличие патентов, разработанных обучающих систем, изданных учебников, наличие у потенциальных разработчиков других методических и инструментальных средств обеспечения учебного процесса. В таких условиях можно использовать универсальный метод ранжирования объектов по множеству количественных показателей [1]. Метод обладает рядом преимуществ. Так, при использовании метода появляется возможность *оперативно выполнять ранжирование (упорядочение) разработчиков по любому подмножеству показателей, одновременно обеспечивая повышение достоверности расчетов; *формировать подгруппы с примерно одинаковыми величинами оценочных показателей, анализировать причины образова- ния таких подгрупп и выявлять взаимосвязи по показателям между претендентами на участие в разработке оценочных материалов; " исключать из дальнейшего рассмотрения тех специалистов, у которых значения конкретных показателей ниже определенного уровня; "осуществлять оптимальный выбор определенной группы разработчиков, располагая сведениями о том, по каким показателям эта группа занимает лидирующее положение; "рассчитывать статистические характеристики (математическое ожидание, дисперсию, коэффициент вариации, медиану, асимметрию, эксцесс), таблицы и гистограммы распределения потенциальных разработчиков в зависимости от численных значений используемых показателей. Кроме того, все расчеты, связанные с обработкой первичной информации и получением результатов, могут выполняться автоматизированно, централизованно.

Предположим, что состав разработчиков определен, множество экзаменационных задач и проблемных ситуаций по специальностям и учебным дисциплинам разработано, сформированы поддерживаемые в актуальном состоянии базы данных, содержащие постоянно пополняемые ${ }^{*}$ множество и специалистов, и " исходное множество разработанных ими задач и проблемных ситуаций.

Теперь из исходного множества задач необходимо выделить искомое подмножество таких задач, которые позволят любой контролирующей системе (преподавателю, экзаменующей комиссии) оценивать степень владения обучаемыми набором заданных образовательным стандартом компетенций. При этом желательно исключить негативное влияние различных внешних и внутренних факторов на результат оценки качества процесса обучения.

Предлагаемый подход к такой оценке базируется на использовании метода пошагового уточнения ранжирования объектов (ПУРО). Метод основан на интеграции дельфийской процедуры прогнозирования будущего (разработан О. Хелмером, Н. Долки, Т. Дж. Гордоном [2; 3]) с предложенной Дж. Кемени (J.G. Kemeny) оценкой медианы и расстояния между упорядочениями объектов [4]. Отличие метода заключается в том, что "дельфийская процедура используется 
при ранжировании экспертами характеристик-функций объектов (а не для прогнозирования будущего); "для количественного анализа степени сходимости мнений экспертов после каждого тура опросов, выявления согласованных групп экспертов и оценки целесообразности завершения экспертизы используется расстояние Кемени (мера близости на отношениях линейного порядка), а в качестве результирующего ранжирования - медиана Кемени; "для группировки участников экспертного опроса, у которых могут оказаться схожими взгляды относительно вариантов ранжирования рассматриваемых характеристик или объектов, устанавливаются пороговые значения расстояния Кемени между ответами экспертов, исходя из вероятности совпадения их мнений, например, близкой к $0.8,0.9,0.95,0.99$; 'для поиска в каждой из образовавшихся групп экспертов согласованного с членами группы ранжирования (упорядочения) характеристик рассчитывается медиана Кемени.

Среди основных преимуществ метода выделим: "повышение точности результатов экспертизы за счет наличия обратной связи при реализации каждого последующего тура; *отсутствие ограничений на количество участников экспертного опроса; "сохранение известных преимуществ и дельфийской процедуры (анонимность и возможность ознакомления с объяснениями, представленными в защиту сильно отличающихся ответов), и алгоритма поиска согласованного упорядочения объектов - корректный расчет медианы и расстояния Кемени; * совместное использование и дельфийской процедуры, и предложенного Кемени корректного подхода к упорядочению функций позволяет получить содержательно приемлемый количественный критерий для обоснованного завершения экспертизы - устанавливается определенная величина относительного (например, 5\%) изменения суммарного расстояния Кемени, а также формировать группы экспертов, ориентируясь на выбранные (в зависимости от степени согласованности мнений) пороговые значения расстояния Кемени и исследовать причины взаимосвязи оценок экспертов, находить согласованное с членами каждой группы экспертов упорядочение характеристик, рассчитав точно или приближенно медиану Кемени. Метод доста- точно широко используется (см., например, [5]) и программно реализован на алгоритмическом языке Python.

Процедура выделения искомого подмножества экзаменационных задач включает следующие шаги.

Шаг 1. Из состава разработчиков оценочных материалов с использованием датчика случайных чисел (ДСЧ) выделяется группа (>10) экспертов.

Шаг 2. Для каждой специальности из множества экзаменационных задач также с использованием ДСЧ выбирают 15 задач для упорядочения по степени (полноте) отражения необходимых компетенций.

Шаг 3. Выполняется ранжирование выделенных задач с использованием метода ПУРО.

Шаг 4. После завершения процесса упорядочения, например, первые 10 задач включают в искомое подмножество.

Процесс пополнения исходного множества экзаменационных задач и реализация процедуры формирования искомого подмножества - базы данных (БД), используемой для *оценки и последующей минимизации затрат времени и финансовых ресурсов на процессы обучения граждан и для "сравнительной оценки потребительского качества методического и инструментального обеспечения учебных процессов, выполняются на регулярной основе.

Наличие БД, созданной с использованием метода ПУРО и содержащей для каждой специальности подмножества экзаменационных задач и проблемных ситуаций, если возникнет необходимость, позволяет выдать требуемое количество задач (используется датчик случайных чисел) по запросу не только представителей вузов, но и обычных граждан.

Но почему оценочные материалы должны быть доступны для любого гражданина? Причина проста и легко объяснима: ведь, во-первых, вероятность ошибки в результатах экзамена обученных граждан (студентов, курсантов, работников, повысивших свою квалификацию и т.д.) обратно пропорциональна мощности множества с задачами и проблемными ситуациями. А во-вторых, постепенно, незаметно, без принуждений будет возрастать количество граждан, умеющих решать собранные в БД сложные (и не очень) 
задачи. Но ведь общество, государство, население страны заинтересованы в том, чтобы росла доля тех, кто может и, возможно, будет пытаться способствовать «улучшающему вмешательству в проблемные ситуации», возникающие в стране.

\section{2. Ранжирование компетенщий и оцен-} ка взаимосвязи межсу дисциплинами при формировании специальностей и учебных планов

1) Метод и инструментарий упорядочения компетенций

При формировании специальностей и состава дисциплин и компетенций возникает необходимость выделения основных компетенций для конкретной специальности, т.е. необходимость упорядочения (ранжирования) выбранных компетенций. Однако, как правило, в реальной ситуации количество возможных компетенций достаточно велико. В таких условиях представляется единственно возможным решением ориентация на метод пошагового уточнения ранжирования объектов (ПУРО). Метод подробно рассмотрен в 3.1 .

2) Метод и инструментарий сравнительной количественной оценки полноты состава и взаимосвязи по компетенциям между учебными дисииплинами одной специальности и между специиальностями

После того как сформированы специальности и определен состав дисциплин по каждой специальности, необходимо убедиться, насколько взаимосвязаны по компетенциям дисциплины и специальности, ведь должны же они хоть как-то отличаться. Да и важно узнать, насколько уникальны специальности, каков информационный вес и компетенции, и специальности и многое другое (см. «Существующие проблемы и трудности», п. 3).

В работе [6] для ответа на перечисленные вопросы использован метод оценки функциональной полноты программных продуктов и других объектов $[7 ; 8]$ и приведен пример применения метода для оценки и улучшения потребительского качества учебного плана путем дополнения его дисциплинами, обеспечивающими формирование у студентов нужных компетенций, заданных образовательным стандартом. Кроме того, показано, что метод позволяет * оценивать вклад, информационный вес каждой темы, модуля, дисциплины, включенной в учебный план, в формирование компетенций, установленных образовательным стандартом по направлениям подготовки специалистов, "выявлять темы, модули, дисциплины, не обеспечивающие получение студентом необходимых знаний, умений и навыков, "обоснованно оценивать качество тестов, формировать или корректировать вариативную часть учебного плана, "проводить сравнительный количественный анализ потребительского качества разработанных в разных вузах учебных планов по конкретным направлениям подготовки специалистов.

Метод программно реализован.

3.3. Экспериментальная оценка времени освоения нового учебного материала (например, разных модулей одной учебной дисциплины и/или сравнительная оценка потребительского качества разных видов и средств обеспечения учебного процесса, например, учебников по одной дисциплине разных авторов и т.д.).

1) Обучаемые - лица примерно одного возраста, одного уровня исходного, базового образования, одинаковой заинтересованности в освоении новой учебной дисциплины, т.е. совокупность достаточно однородных объектов.

Использованный метод рассмотрим на конкретном примере. Эксперимент проводился нами в течение нескольких лет и состоял в оперативном выполнении студентами трех групп одной специальности (60-70 человек) учебного задания - в решении конкретной задачи. Задание выполнялось после прослушивания нового теоретического материала, т.е. сначала излагался теоретический материал, а затем студенты получали задание, выполнить которое могли только те, кто хорошо освоил теоретический материал, прослушанный вначале, на первом часе лекции (напомним, что «Знание - проверенный практикой результат познания действительности, верное отражение ее в сознании человека» (БСЭ)). Содержательная формулировка задачи была одинаковой у всех студентов, разными были только численные значения исходных данных. Время, отводимое на выполнение задания, - один академический час (45 мин.). Стимул для быстрого решения задачи - решивший сдает листки с решени- 
ем и может покинуть аудиторию. Оказалось, что отдельные студенты решали задачи менее чем за двадцать минут, другие не справлялись с заданием даже в течение двух академических часов, т.е. при одинаковых внешних условиях и одинаковом исходном уровне знаний теоретических положений в очередной раз подтверждено очень существенное влияние индивидуальных способностей на скорость осознания возможностей практического применения полученных знаний и реальной реализации этих возможностей. В результате эксперимента установлено, что время, достаточное для практического освоения нового учебного материала, имеет существенный разброс, значительную величину правосторонней асимметрии и зачастую может быть аппроксимировано альфа-распределением. Позднее экспериментально подтверждена гипотеза о том, что при одинаковом уровне начальной подготовки и условиях эксперимента с увеличением сложности работы должна возрастать и величина правосторонней асимметрии распределения затрат времени на выполнение работы.

Предположим, что необходимо сравнить характеристики потребительского качества двух учебников У1 и У2 (разных авторов). Требуется оценить разницу в затратах времени обучаемых на подготовку к экзамену (разницу в затратах времени на владение заданными образовательным стандартом компетенциями) по специальной учебной дисциплине при использовании У1 и У2. В случае отсутствия такой разницы сравнить цены учебников, стоимость доставки и другие характеристики потребительского качества.

При использовании натурного эксперимента рассматриваемая методика предполагает (применительно к сравнительной оценке характеристик потребительского качества различных видов и средств методического и инструментального обеспечения процессов обучения студентов) реализацию следующих шагов.

Шаг 1. Студентам групп Г1 и Г2 предлагают при подготовке к экзамену использовать учебник У1, а студентам групп Г3 и Г4 учебник У2.

Шаг 2. Во время экзамена преподаватель фиксирует затраты времени каждого ученика на решение установленного набора задач и проблемных ситуаций (или количество решенных задач за установленное время).

Шаг 3. Выполняется обработка результатов экзамена в четырех группах: разделение на децили, выделение интердецильного размаха, оценка статистических характеристик распределения интердецильного размаха.

Шаг 4. Выполняется проверка на однородность выборок (интердецильных размахов в каждой из групп Г1, Г2 и Г3, Г4) с использованием критерия Колмогорова и Смирнова $[9 ; 10]$. Если нулевая гипотеза не отвергнута в каждом из двух случаев, то эти группы объединяют, т.е. Г1 с Г2, а Г3 с Г4 (объединяют выборки - интердецильные размахи).

Шаг 5. Выполняется сравнение на однородность по критерию Колмогорова и Смирнова объединенных выборок - Г1 + Г2 (использован при подготовке к экзамену учебник У1) и Г3 + Г4 (использован учебник У2).

Допустим, что нулевая гипотеза отвергнута при выбранной доверительной вероятности - выборки из разных генеральных совокупностей (т.е. оказалось, что подготовка к экзамену с использованием одного из учебников более предпочтительна).

Шаг 6. Выполняется оценка статистических характеристик распределения выборок Г1 + Г2 и Г3 + Г4, расчет доверительных границ для средних значений затрат времени в каждой выборке, анализ причин попадания студентов в первый и девятый децили, с использованием инструментальных средств имитационного моделирования определяют общее для всех $m$ групп распределение затрат времени на освоение нужных компетенций (как среднее $m$ случайных величин с известным, полученным в результате имитационного моделирования распределением в каждой из $m$ групп), выполняют сравнение цен на У1 и У2 и т.д.

[Обратим внимание на то, что при проведении описанного ранее в качестве примера эксперимента у всех студентов были одинаковыми внешние мотивационные стимулы, условия проведения эксперимента и сложность очередного осваиваемого материала (в процессе изучения разных тем учебной дисциплины сложность осваиваемого материала постоянно изменялась - участникам предлагались разные по сложности темы учебного курса и задачи). Соответственно, 
влияние на дисперсию и другие статистические характеристики времени освоения всех тем учебной дисциплины оказывали индивидуальные способности обучаемого, сложность теоретического материала и личная мотивация (личные приоритеты мотивационных стимулов). Поэтому по результатам имитационного моделирования нельзя оценить, какая часть дисперсии или коэффициента вариации времени освоения учебной дисциплины связана с индивидуальными способностями, а какая - со сложностью изученного материала.]

2) Обучаемье - лица разного возраста, разного уровня образования, разной степени заинтересованности в результатах обучения и с разныли психофизиологическими и физическими особенностями.

Поведение любого индивида носит вероятностный характер. Это относится и к любой деятельности индивида, включая затраты времени на освоение любого нового учебного материала. Так, затраты времени на выполнение одинаковых операций делового процесса существенно отличаются не только у разных исполнителей, но даже у одного исполнителя, выполняющего одну операцию, затраты времени на ее выполнение имеют значительный разброс, и значения коэффициента вариации и правосторонней асимметрии распределения могут быть весьма заметными. Экспериментальную оценку статистических характеристик распределения затрат времени на изучение методических рекомендаций, учебных материалов, связанных с темой повышения квалификации, руководства пользователя и т.д., необходимо "проводить, группируя обучаемых (пользователей, потенциальных клиентов-покупателей, слушателей курсов повышения квалификации) в зависимости от значений классификационных признаков (пол, возраст, образование и др.), и *выполнять имитационное моделирование с учетом характеристик распределения затрат времени в каждой из групп и доли конкретной группы в составе обучаемых.

Bblвoдbl. Предложенные методы обработки результатов экспериментальной оценки характеристик потребительского качества видов и средств методического и инструментального обеспечения учебных процессов, оценки распределения времени освоения мо- дулей учебной дисциплины (т.е. оценки результатов практического использования полученных знаний) позволяют:

1) количественно оценивать вероятность того, что времени, отведенного учебным планом на изучение конкретной дисциплины, будет достаточно для обеспечения требуемого качества образовательных услуг, т.е. количественно оценивать долю учащихся, обладающих необходимыми знаниями, умениями, навыками, компетенциями;

2) проводить сравнительный анализ (с использованием методов непараметрической статистики): а) скорости освоения потенциальными потребителями содержания * вариантов руководства пользователя и методических рекомендаций по решению задач, *проектов законов и поправок к ним, "вариантов различных нормативных материалов, инструкций и учебных пособий; б) качества обучения разными преподавателями, в разных учебных группах;

3) с большей достоверностью оценивать влияние различных учебно-методических инструментов на качество обучения.

3.4. Формирование учебного плана: экспертная оценка времени, необходимого для изучения учебной дисциплины. Обеспечить достоверность оценки характеристик распределения затрат времени обучаемых на изучение нового материала путем экспериментальных исследований не всегда удается. Часто в этих целях используются экспертные методы. При этом в процессе экспертизы желательно максимально стимулировать активную интеллектуальную деятельность участников экспертной группы, исключить влияние личных качеств участников на результаты экспертизы, корректно обобщить суждения всех членов экспертной группы. Особенность предлагаемого подхода состоит, во-первых, в использовании многошаговой процедуры, на каждом шаге которой осуществляется имитационное моделирование, и, во-вторых, в интеграции метода Дельфи c экспертизой, направленной на получение обобщенного мнения группы экспертов о возможном диапазоне значений искомого показателя. Такое объединение дает ряд преимуществ, в частности, предоставляет специалистам, участвующим в экспертизе, возможность рассматривать возражения и пред- 
ложения других членов экспертной группы в атмосфере, свободной от влияния личных качеств участников. Одновременно появляется возможность использовать так называемое «информированное интуитивное суждение» специалиста-эксперта путем создания таких условий, когда эксперт может активно взаимодействовать с другими специалистами в этой области или в областях, касающихся прочих аспектов изучаемой проблемы. При этом непосредственное общение специалистов друг с другом заменяется последовательностью шагов, на каждом из которых реализуется полный цикл экспертизы, включая информирование специалистов-экспертов о результатах предыдущего шага.

Предположим теперь, что описанная интеграция с методом Дельфи реализована. Но, спрашивается, как определить, что коллективное мнение стабилизировалось и пора прекращать дальнейшие опросы? С какой вероятностью, например, не будет превышено определенное значение искомого показателя? Какова вероятность того, что значение показателя будет находиться в заданных доверительных границах? Перечень подобных вопросов может быть продолжен.

Алгоритм оценки. Для ответа на поставленные вопросы, по-видимому, единственно обоснованной процедурой является предлагаемая ниже последовательность шагов (см. также $[5 ; 11 ; 12])$.

Шаг 1. Оценки каждого $i$-го эксперта на $j$-м шаге $T^{(j)}{ }_{\text {i }}$ (минимальное, максимальное и наиболее вероятное значения показателя) представляются в виде треугольного или равномерного (если указаны только два значения затрат времени на освоение учебной дисциплины) распределений.

Шаг 2. Обобщенное коллективное мнение $n$ экспертов об искомом значении анализируемого показателя определяется как среднее $n$ случайных величин, имеющих треугольное или равномерное распределения (мнений $n$ участников экспертной группы) путем реализации на каждом $k$-м шаге имитационного моделирования.

Шаг 3. В результате имитационного моделирования на каждом $k$-м шаге получают статистические характеристики (математическое ожидание, дисперсию, коэффициент вариации, эксцесс, асимметрию) и распределение (в фор- ме гистограммы и таблицы) значений искомого показателя - функции $T^{(k)}{ }_{\text {об }}=\mathrm{f}\left(T^{(k)}{ }_{i}\right)$.

Шаг 4. После каждого шага (цикла экспертизы) участников экспертной группы знакомят с объяснениями, представленными в защиту сильно отличающихся оценок анализируемого показателя, и предлагают при желании изменить свои предыдущие ответы.

Шаг 5. На каждом очередном $j$-м шаге оценивают изменение значений коэффициента вариации $K^{(j)}{ }_{v a r}$ функции $T^{(j)}{ }_{\text {об. }}$. При отклонении коэффициента вариации от предыдущего значения, например, на $5 \%$ и менее можно считать, что оценки экспертов стабилизировались и целесообразно завершать экспертизу, т.е. если $\left|K^{(j)}{ }_{v a r}-K^{(j+l)}{ }_{v a r}\right| * 100 / K^{(j)}{ }_{v a r \%}<5 \%$, то можно завершать экспертизу.

Шаг 6. На основании результатов имитационного моделирования на последнем шаге оценивают доверительные границы значений искомого показателя и вероятность того, что его значения окажутся больше или меньше определенного числа.

Заключение. В результате проведенных исследований впервые:

1. Создана универсальная методика оценки затрат времени и финансовых ресурсов на обучение граждан; выявлены и систематизированы проблемы и трудности, препятствующие оптимальной организации учебных процессов, отмечено, что в настоящее время отсутствуют обязательные в масштабах всей страны, единые для всех (обучаемых и преподавателей) варианты контрольных испытаний - экзаменационных задач, перечни проблемных ситуаций, невозможно определить полноту охвата содержанием дисциплин всей совокупности компетенций, оценить, в какой степени десятки включенных в учебный план дисциплин обеспечивают возможность получения обучаемым множества необходимых компетенций, и какими дисциплинами такие возможности лучше обеспечиваются, а какими - хуже, и насколько.

2. Показано, что разное потребительское качество отдельных видов и средств методического, кадрового и инструментального обеспечения учебного процесса, разная их цена и степень влияния на величину затрат времени и финансовых ресурсов на обучение граждан одновременно приводит 
и к разной результативности учебного процесса, к тому, что выпускники одинаковых специальностей, но разных вузов, в разных городах одной страны, в разных административно-территориальных образованиях имеют разный уровень (объем) знаний, умений, навыков, компетенций.

3. Обоснованы исходные условия и критерии оценки качества обучения граждан, отмечено, что использование интердецильного размаха, практически не зависящего от экстремальных значений в выборке (появляется возможность исключить или уменьшить влияние неоднородности в составе обучаемых) и позволяющего иметь очень малые колебания стандартного отклонения в выборках из одной и той же генеральной совокупности, дает возможность, выполняя оценку потребительского качества методического и инструментального обеспечения учебных процессов, допускать применение треугольного распределения при имитационном моделировании, а в качестве критерия оценки однородности ориентироваться на применение наиболее строгого критерия однородности: критерия Колмогорова и Смирнова.

4. Предложена совокупность методов и инструментальных средств *для формирования состава разработчиков оценочных материалов, используемых *для оценки наличия у обучаемого определенного (заданного) уровня знаний, *для ранжирования и обоснованного выбора состава компетенций при формировании специальностей и учебных планов, "для экспериментальной сравнительной оценки затрат времени обучаемых на изучение новых материалов и для оценки потребительского качества разных видов и средств обеспечения учебного процесса, * для экспертной оценки необходимого и достаточного времени на изучение дисциплин, представленных в учебных планах специальностей.

\section{Список источников}

1. Хубаев Г.Н. Ранжирование объектов по множеству количественных показателей: универсальный алгоритм // РИСК: Ресурсы, Информация, Снабжение, Конкуренция. 2018. №1. C. 213-217.
2. Хелмер О. Анализ будущего: метод Дельфи // Научно-техническое прогнозирование для промышленности и правительственных учреждений / Пер. с англ. Под ред. Г. М. Доброва. М.: «Прогресс», 1972. С. 77-83.

3. Helmer O. Social Technology. New York, Basic Books, Inc., Publishers, 1966.

4. Kemeny J.G. Generalized random variables. Pacific Journal of Mathematics. 1959. Vol. 9. Pp. 1179-1189.

5. Родина О.В. Налоговый учет: экономико-математические модели, методы и программные средства для оценки и минимизации затрат ресурсов на ведение и мониторинг: монография. М.: ФГБОУ ВПО «РЭУ им. Г. В. Плеханова», 2011. 144 с.

6. Хубаев Г.Н. Количественная оценка потребительского качества учебных планов по направлениям подготовки специалистов // Проблемы экономики. 2012. №3. С. 36-41.

7. Курбесов А.В. Математические и имитационные модели для оценки качества и оптимизации информационного обеспечения системы управления обязательным медицинским страхованием: автореф. дис. ... канд. экон. наук. Ростов-на-Дону, 2000. 26 с.

8. Щербаков С.М. Экономико-математическое моделирование интернет-приложений: монография. Ростов-на-Дону: РГЭУ (РИНХ), 2010. 165 c.

9. Колмогоров А.Н. Теория вероятностей и математическая статистика. М.: Наука, 1986. C. 134-141.

10. Kolmogoroff A. N. Sulla determinazione empirica di una legge di distribuzione. Giornale Istituto Italiano. Attuare 4 (1933). P. 83-91.

11. Широбокова С.Н., Щербаков С.М. Метод и программная система имитационного моделирования на основе языка UML как инструмент анализа и моделирования деловых процессов // Вопросы современной науки и практики. Университет им. В.И. Вернадского. 2008. Т. 2. №4. C. 154-161.

12. Родина О. В., Калугян К. Х. Экспрессоценка и оптимизация затрат ресурсов на освоение учебных дисциплин // Сетевое партнерство в науке, промышленности и образовании (NCSIE): Труды Международной мультиконференции (Санкт-Петербург, СПбПУ Петра Великого, 4-6 июля 2016 г.). Санкт-Петербург: СПбПУ, 2016. С. 75-84. 


\section{References}

1. Hubaev G. N. Ranzhirovanie ob'ektov po mnozhestvu kolichestvennyh pokazatelej: universal'nyj algoritm [Ranking of objects by a set of quantitative indicators: a universal algorithm]. RISK: Resursy, Informacija, Snabzhenie, Konkurencija [RISC: Resources, Information, Supply, Competition]. 2018; 1: 213-217. (In Russ.).

2. Helmer O. Analiz budushhego: metod Del'fi [Analysis of the future: the Delphi method]. Nauchno-tehnicheskoe prognozirovanie dlja promyshlennosti i pravitel'stvennyh uchrezhdenij [Scientific and technical forecasting for industry and government agencies]. Per. $\mathrm{s}$ angl. Pod red. G.M. Dobrova [Trans. from English. In G. M. Dobrov (eds.)]. Moscow: «Progress», 1972. Pp. 77-83. (In Russ.).

3. Helmer O. Social Technology. New York, Basic Books, Inc., Publishers, 1966.

4. Kemeny J.G. Generalized random variables. Pacific Journal of Mathematics. 1959. Vol. 9. Pp. 1179-1189.

5. Rodina O.V. Nalogovyj uchet: jekonomiko-matematicheskie modeli, metody i programmnye sredstva dlja ocenki i minimizacii zatrat resursov na vedenie i monitoring: monografija [Tax accounting: economic and mathematical models, methods and software tools for assessing and minimizing resource costs for management and monitoring: monograph]. Moscow: FGBOU VPO «RJeU im. G. V. Plehanova», 2011. 144 p. (In Russ.).

6. Hubaev G.N. Kolichestvennaja ocenka potrebitel'skogo kachestva uchebnyh planov po napravlenijam podgotovki specialistov [Quantitative assessment of the consumer quality of curricula in the areas of training specialists]. Problemy jekonomiki [Problems of Economics]. 2012; 3: 36-41. (In Russ.).

7. Kurbesov A.V. Matematicheskie i imitacionnye modeli dlja ocenki kachestva i optimizacii informacionnogo obespechenija sistemy upravlenija objazatel'nym medicinskim strahovaniem: avtoref. dis. ... kand. jekon. nauk [Mathematical and simulation models for assessing the quality and optimization of information support for the mandatory health insurance management system: Ph. D. (Economic) Thesis]. Rostov-on-Don, 2000.26p. (In Russ.).

8. Shherbakov S.M. Jekonomiko-matematicheskoe modelirovanie internet-prilozhenij: monografija [Economic and mathematical modeling of Internet applications: monograph]. Rostov-on-Don: RGJeU (RINH), 2010. 165 p. (In Russ.).

9. Kolmogorov A. N. Teorija verojatnostej i matematicheskaja statistika [Probability theory and mathematical statistics]. Moscow: Nauka, 1986. Pp. 134-141. (In Russ.).

10. Kolmogoroff A.N. Sulla determinazione empirica di una legge di distribuzione. Giornale Istituto Italiano. Attuare 4 (1933). P. 83-91.

11. Shirobokova S.N., Shherbakov S.M. Metod i programmnaja sistema imitacionnogo modelirovanija na osnove jazyka UML kak instrument analiza i modelirovanija delovyh processov [Method and software system of simulation modeling based on the UML language as a tool for analysis and modeling of business processes] // Voprosy sovremennoj nauki i praktiki. Universitet im. V.I. Vernadskogo [Issues of modern science and practice. V.I. Vernadsky University]. 2008; 2 (4): 154-161. (In Russ.).

12. Rodina O. V., Kalugjan K.H. Jekspressocenka i optimizacija zatrat resursov na osvoenie uchebnyh disciplin [Express assessment and optimization of resource costs for the development of academic disciplines]. Setevoe partnerstvo v nauke, promyshlennosti i obrazovanii (NCSIE): Trudy Mezhdunarodnoj mul'tikonferencii (Sankt-Peterburg, SPbPU Petra Velikogo, 4-6 ijulja 2016 g.) [Network Partnership in Science, Industry and Education (NCSIE): Proceedings of the International Multi-Conference (St. Petersburg, Peter the Great SPbPU, July 4-6, 2016)]. Saint Petersburg: SPbPU, 2016. Pp. 7584. (In Russ.).

Статья поступила в редакцию 02.09.2021; одобрена после рецензирования 10.09.2021; принята к публикации 22.09.2021.

The article was submitted on 02.09.2021; approved after reviewing on 10.09.2021; accepted for publication on 22.09.2021. 


\section{ИНФОРМАЦИЯ ОБ АВТОРАХ}

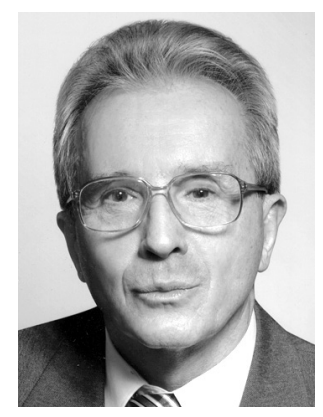

Хубаев Георгий Николаевич - доктор экономических наук, кандидат технических наук, профессор, профессор кафедры «Информационные системы и прикладная информатика», Ростовский государственный экономический университет (РИНХ).

Россия, г. Ростов-на-Дону, ул. Б. Садовая, 69

Georgy N. Khubaev - Doctor of Economic Sciences, Candidate of Engineering Sciences, Professor, Professor of the Department of Information Systems and Applied Informatics, Rostov State University of Economics (RSUE).

69 B. Sadovaya st., Rostov-on-Don, Russia 